\title{
Table Structure Extraction from Form Documents Based on Gradient-Wavelet Scheme
}

\author{
Dihua Xi and Seong-Whan Lee \\ Center for Artificial Vision Research, Korea University \\ Anam-dong, Seongbuk-ku, Seoul 136-701, Korea \\ $\{$ dhxi, swlee\}@image.korea.ac.kr
}

\begin{abstract}
Based on gradient and wavelet analyses, a novel scheme has been developed to extract table structures from skewed form document images. In this scheme, first, a skewed form document image is rotated according to the angle obtained from the gradient algorithm. Then the deskewed image is decomposed into four sub-images by divisible Multiresolution Analysis(MRA) wavelets. Afterwards, the table structure image which represents the geometric structure of the form can be obtained from the sub-images by a modified wavelet reconstruction algorithm. Meanwhile, another document image without table lines can be produced by Minkowski operation and is referred to as a table free image. Experimental results indicate that this new scheme can be applied to process the skewed form document images with promising achievements.
\end{abstract}

\section{Introduction}

A knowledge acquisition bottleneck has become the major barrier to the development and implementation of effective information systems. To mitigate this bottleneck, document processing techniques should be considered to automatically acquire information from various types of documents.

Form document processing is an essential operation in many business and government organizations. Particularly, in financial organizations, millions of financial transactions take place every day. They are associated with conventional form documents such as bank cheques, payment slips, bills, etc. However, there are a number of limitations with current manual operations of the form documents. These include, for example, the cost of information acquisition from printed forms, delay in accessing the stored forms, vulnerability of the stored forms to loss, damage, etc. The cost of information capture from the printed forms can be very high.

For large corporations and government organizations, the cost can be several orders of magnitude[1]. Thus, any development which can improve the processing of such documents will make a significant contribution. A lot of researchers have paid attention to the development of many methods to solve this problem, and some achievements can be found in $[1,2,3]$.

The form documents may contain tables, and most of significant information is sited in these tables. Therefore, how to extract geometric structures plays an 
intrinsic role in document analysis. Particularly, the table structure is the main class of the geometric structures in the form documents. And form document image usually has a little skew and slant. This paper presents a gradient based approach for skew correction and a wavelet based approach for table extraction from the form document images. The basic idea is described as the following steps:

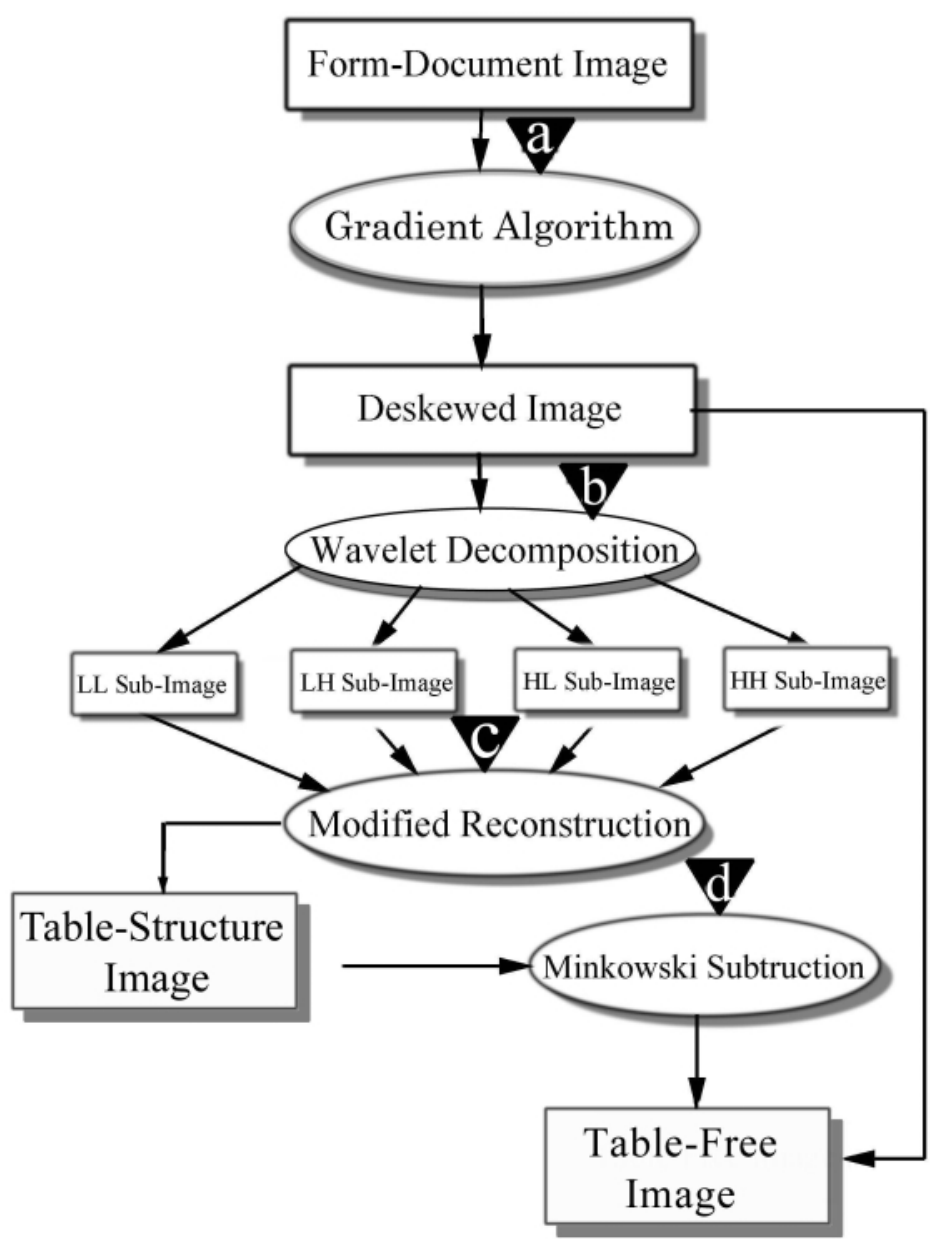

Fig. 1. Diagram of the gradient wavelet scheme for form document analysis.

- Gradient operation is performed on the form document image to evaluate the skew angle. Afterwards, a deskewed image can be obtained by rotating the original form document image. 
- The deskewed image is decomposed into four sub-images [4] by the wavelet decomposition algorithm [5].

- According to some of these sub-images, we can reconstruct an image that contains only horizontal and vertical lines from which a particular image can be composed, and is referred to as table structure image. Therefore, the table structure of the form document image can be obtained. For this purpose, a modified wavelet reconstruction algorithm will be developed in this paper.

- Using the table structure image and the deskewed image, an image which does not contain table lines is constructed by the Minkowski subtraction [6], and is referred to as table free image. Furthermore, the important information position can be given by the form structure.

In summary, the form document image is first deskewed. Then, the image is decomposed into sub-images. Afterwards, the table structure and the table free image are reconstructed according to wavelet theory and Minkowski operation respectively. Thus, in this paper, the gradient and wavelet analysis play major roles. This is the reason why this new method is called gradient-wavelet scheme. The overall diagram of this scheme is illustrated in Fig. 1, where four main operations in the scheme are described as

a) Skew Correction: a digital form image represented by function $f(m, n)$ is captured from a form document by optical scanning. Afterwards, based on the gradient algorithm, we can evaluate the rotational angle and deskew the form document image.

b) Wavelet Decomposition: based on the wavelet decomposition, the image is decomposed into four sub-images which are referred to as LL, LH, HL and $\mathrm{HH}$ sub-images respectively.

c) Modified Wavelet Reconstruction: the table structure image can be obtained by the modified wavelet reconstruction algorithm.

d) Minkowski Subtraction: according to the table structure image and the deskewed image, the table free image can be obtained by the algorithm of the picture difference.

\section{Skew Correction}

The form document images are obtained through scanning and binarization. The skew correction steps include:

- Noise removal: because scanned images are usually not clean, we used the median filter to remove noise.

- Deskewing: for an image described by $\mathrm{f}(\mathrm{x}, \mathrm{y})$, the gradient vector $[p, q]^{T}$ is defined by

$$
p=\frac{\partial f(x, y)}{\partial x}, \quad q=\frac{\partial f(x, y)}{\partial y} .
$$


The orientation of this gradient vector is

$$
\varphi=\arctan (q / p)
$$

For every black point $(x, y)$ in the image, its gradient orientation $\left.\varphi_{(} x, y\right)$ can be computed or calculated by Eq. (1), Eq. (2), and Eq. (3). Suppose that the region used to computed or calculated $\varphi(x, y)$ is $[x-N, x+N] \times[y-N, y+N]$ and it contains $\mathrm{M}$ black points. We have

$$
\begin{gathered}
\left\{\begin{array}{l}
\bar{x}=\frac{1}{M} \sum_{i=-N}^{N} \sum_{j=-N}^{N}(x+i), \quad \text { if } f(x+i, y+j) \text { is black } \\
\bar{y}=\frac{1}{M} \sum_{i=-N}^{N} \sum_{j=-N}^{N}(y+j), \quad \text { if } f(x+i, y+j) \text { is black }
\end{array}\right. \\
\mu_{p, q}=\sum_{i=-N}^{N} \sum_{j=-N}^{N}(x+i-\bar{x})^{p}(y+j-\bar{y})^{q}, \quad \text { if } f(x+i, y+j) \text { is black, } \\
\varphi(x, y)=\frac{1}{2} \arctan \left(\frac{2 * \mu_{1,1}}{\mu_{2,0}-\mu_{0,2}}\right) .
\end{gathered}
$$

According to above equations, the gradient orientation can be obtained by using $(2 N+1) \times(2 N+1)$ (in our experiment, $\mathrm{N}=3$ ) filter operation. The range of $\varphi(x, y)$ is $\left[-\frac{\pi}{2}, \frac{\pi}{2}\right)$. Because the orientations of two different points on the table lines are parallel or orthogonal, the range of $\varphi(x, y)$ can be transformed into $\left(-\frac{\pi}{4}, \frac{\pi}{4}\right)$ by

$$
\phi(x, y)= \begin{cases}\varphi(x, y), & \text { if }|\varphi|<\frac{\pi}{4} \\ -\operatorname{sign}(\varphi)\left(\frac{\pi}{2}-|\varphi|\right), & \text { if }|\varphi|>\frac{\pi}{4}\end{cases}
$$

where $\operatorname{sign}(\varphi)$ is the sign function,

$$
\operatorname{sign}(x)=\left\{\begin{array}{cl}
1, & \text { if } x>0 \\
0, & \text { if } x=0 \\
-1, & \text { if } x<0
\end{array}\right.
$$

For detecting the orientation of a skewed form document, a half of the range of $\varphi$ will be enough. If we have some knowledge about the range of the skew angle, we can reduce the domain of $\varphi$ even further.

The gradient algorithm is based on the observation of the orientation distribution of all black points in the image. For a skewed form-document image, there will be much more points in the image whose gradient orientations are parallel or perpendicular to the direction of a table line direction. In fact, all points on the table lines have such characteristics. It is obvious to consider that statistical information can be used for skew angle detection. Fig. 2 gives an example shape of the gradient orientation histogram of an form-document image. Fig. 2 (a) shows an original image of the skewed document, and (b) gives the histogram of point distribution on different orientations. 


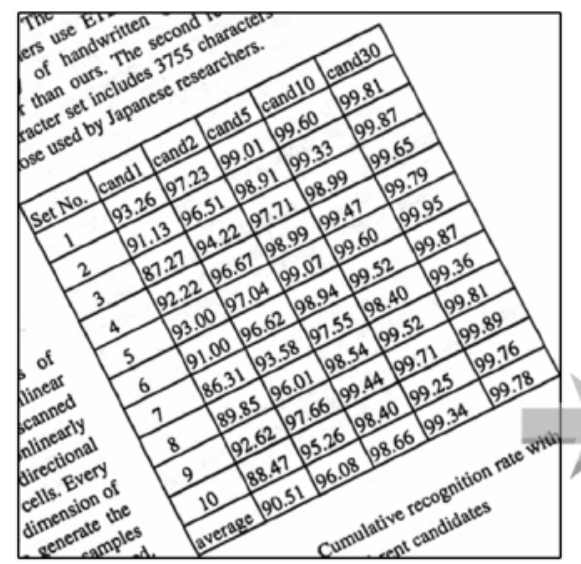

(a)

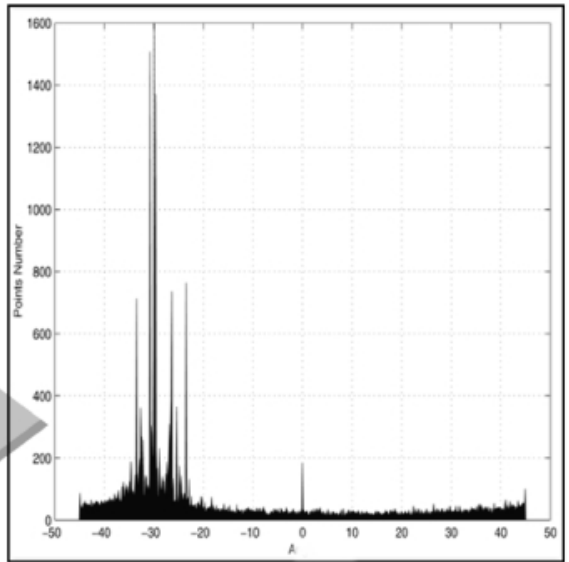

(b)

Fig. 2. Example to obtain the skewed angle based on the points distribution histogram on different orientations. (a) Original image of skewed form document. (b) The distribution histogram on different gradient orientations of the original image.

Based on the points distribution histogram on different orientations, the gradient algorithm searches for the maximum in the histogram to obtain the skew angle (in Fig. 2, the angle is -29 degrees). By using this skew angle $\theta$, the skewed form document image is aligned by the rotation transformation

$$
\left\{\begin{array}{l}
x^{\prime}=x \cos \theta+y \sin \theta \\
y^{\prime}=-x \sin \theta+y \cos \theta
\end{array},\right.
$$

where $(x, y)$ is a coordinate before deskewing, and $\left(x^{\prime}, y^{\prime}\right)$ is the coordinate after deskewing.

\section{Decomposition of Form Document by MRA Wavelet}

In this section, the basic concepts of Multiresolution Analysis(MRA) wavelet will be introduced and followed by a description of how the MRA wavelet can be used to decompose the form documents.

\subsection{Basic Concepts of MRA Wavelet}

Let $\left\{V_{j}\right\}_{j \in Z}$ be a closed subset of $L^{2}(R)$. It is known that $\left\{V_{j}^{2}\right\}_{j \in Z}$ is a multiresolution of $L^{2}\left(R^{2}\right)$ if and only if $\left\{V_{j}\right\}_{j \in Z}$ is a multiresolution of $L^{2}(R)$, and

$$
V_{j}^{2}=V_{j} \otimes V_{j}
$$


Let $\varphi(x)$ be a scaling function of $\left\{V_{j}\right\}_{j \in Z}$. Therefore, $\Phi(x, y)=\varphi(x) \varphi(y)$ is a scaling function of $\left\{V_{j}^{2}\right\}_{j \in Z}$. For every $j \in Z$, the function system

$$
\left\{\Phi_{j, k_{1}, k_{2}}=\varphi_{j, k_{1}}(x) \varphi_{j, k_{2}}(x) \mid\left(k_{1}, k_{2}\right) \in Z^{2}\right\}
$$

becomes an orthonormal basis of $V_{j}^{2}$. Such multiresolution analysis is called divisible MRA.

We can define a wavelet space $W_{j}^{2}=\left(V_{j}^{2}\right)^{\perp}$, namely

$$
W_{j}^{2} \oplus V_{j}^{2}=V_{j-1}^{2}
$$

From the above analysis, we can easily produce a very significant formula:

$$
\begin{aligned}
V_{j-1}^{2} & =V_{j-1} \otimes V_{j-1} \\
& =\left(W_{j} \oplus V_{j}\right) \otimes\left(W_{j} \oplus V_{j}\right) \\
& =\left(W_{j} \otimes W_{j}\right) \oplus\left(V_{j} \otimes W_{j}\right) \oplus\left(W_{j} \otimes V_{j}\right) \oplus\left(V_{j} \otimes V_{j}\right) .
\end{aligned}
$$

For one-dimensional MRA, because $\varphi(x)$ is the scaling function of $\left\{V_{j}\right\}_{j \in Z}$, we can produce a wavelet function

$$
\psi(x)=\sum_{k=-\infty}^{+\infty} g_{k} \varphi_{-1, k}(x)=2^{-\frac{1}{2}} \sum_{k=-\infty}^{+\infty} g_{k} \varphi(2 x-k)
$$

such that for every $j \in Z$, the function system

$$
\left\{\psi_{j, k}(x)=2^{-\frac{j}{2}} \psi\left(2^{-j} x-k\right) \mid k \in Z\right\}
$$

be the orthonormal bases of $W_{j}$.

Similar to the one-dimensional MRA, three basic wavelet functions $\psi^{1}, \psi^{2}, \psi^{3}$ can be produced from the two-dimensional MRA, and described as

$$
\left\{\begin{array}{l}
\psi^{1}(x, y)=\psi(x) \varphi(y) \\
\psi^{2}(x, y)=\varphi(x) \psi(y) \\
\psi^{3}(x, y)=\psi(x) \psi(y)
\end{array}\right.
$$

The orthonormal bases of the wavelet space $W_{j}^{2}$ can be obtained from these three single wavelet functions $\psi^{1}, \psi^{2}, \psi^{3}$ by a dilation $\left(2^{j}\right)$ and a dyadic translation (of $\frac{k}{2^{j}}$ ).

\subsection{Wavelet Decomposition for Sub-images}

The form document image $f(m, n)$ is transformed into four sub-images by the wavelet decomposition. These sub-images are represented by $f_{L L}(m, n), f_{L H}(m, n), f_{H L}(m, n)$, and $f_{H H}(m, n)$ [4]. Mathematically, they are described by 


$$
\left\{\begin{array}{l}
f_{L L}\left(k_{1}, k_{2}\right)=\sum_{m_{1}=0}^{2 N-1} \sum_{m_{2}=0}^{2 N-1} h_{m_{1}} h_{m_{2}} f\left(m_{1}+2 k_{1}, m_{2}+2 k_{2}\right) \\
f_{L H}\left(k_{1}, k_{2}\right)=\sum_{m_{1}=0}^{2 N-1} \sum_{m_{2}=0}^{2 N-1} h_{m_{1}} g_{m_{2}} f\left(m_{1}+2 k_{1}, m_{2}+2 k_{2}\right) \\
f_{H L}\left(k_{1}, k_{2}\right)=\sum_{m_{1}=0}^{2 N-1} \sum_{m_{2}=0}^{2 N-1} g_{m_{1}} h_{m_{2}} f\left(m_{1}+2 k_{1}, m_{2}+2 k_{2}\right) \\
f_{H H}\left(k_{1}, k_{2}\right)=\sum_{m_{1}=0}^{2 N-1} \sum_{m_{2}=0}^{2 N-1} g_{m_{1}} g_{m_{2}} f\left(m_{1}+2 k_{1}, m_{2}+2 k_{2}\right)
\end{array}\right.
$$

where $\left\{h_{k}\right\}$ denotes a frequency response which depends on the used wavelet function, and,

$$
g_{k}=(-1)^{2 N-k+1} h_{2 N-k-1} .
$$

In Eq. (10), for a given integer $N$ and a function $f(m, n)$, four functions of the sub-images can be derived. Clearly, the computational complexity of the algorithm increases as $N$ increases. If $N$ is too small, the filtering effect will be dropped. Therefore, we should choose a suitable value for $N$ in our study. Experiments have shown that the results are acceptable when $N=3$ or $N=4$. In this paper, $N=3$ has been chosen for all experiments.

The document image can be regarded as $V_{j-1}^{2}$. According to Eq. (7), $V_{j-1}^{2}$ can be represented by

$$
V_{j-1}^{2}=\left(W_{j} \otimes W_{j}\right) \oplus\left(V_{j} \otimes W_{j}\right) \oplus\left(W_{j} \otimes V_{j}\right) \oplus\left(V_{j} \otimes V_{j}\right) .
$$

Therefore, the form document image $f(m, n)$ can be divided into four subimages, namely,

$$
f(m, n)=f_{L L} \oplus f_{L H} \oplus f_{H L} \oplus f_{H H}
$$

where $f_{L L}, f_{L H}, f_{H L}$ and $f_{H H}$ denote LL sub-image, LH sub-image, HL subimage and $\mathrm{HH}$ sub-image respectively, and can be computed by Eq. (10). The characteristics of these sub-images are described as follows:

- LL sub-image: both horizontal and vertical directions are low-frequency. It corresponds to $V_{j} \otimes V_{j}$.

- LH sub-image: the horizontal direction is low-frequency and the vertical direction is high-frequency. It corresponds to $V_{j} \otimes W_{j}$

- HL sub-image: the horizontal direction is high-frequency and the vertical direction is low-frequency. It corresponds to $W_{j} \otimes V_{j}$.

- HH sub-image: both horizontal and vertical directions are high-frequency. It corresponds to $W_{j} \otimes W_{j}$.

It is obvious that the LL sub-image has a "smoothing" effect in both directions. The LH sub-image is the result from a filter in which the image has been smoothed in horizontal direction and enhanced in vertical direction. The HL 
sub-image is the result from a filter in which the image has been enhanced in horizontal direction and smoothed in vertical direction. The HH sub-image has an "enhancing" effect in both directions. Fig. 3 shows the above characteristics of these four sub-images. The original gray level image is shown in Fig. 3(a). Figs. 3(b)-(e) are its sub-images decomposed by Daubechies wavelet. It can be shown that the LH sub-image contains only the lines in horizontal direction, while the HL sub-image contains only the lines in vertical direction. From this example, it can be observed that the horizontal and vertical lines of the image can be extracted from the LH and HL sub-images. Afterwards, the tables which are composed of the horizontal and vertical lines in the form documents can be obtained. Another example is depicted in Fig. 4.

\section{Reconstruction of Table Structure Image and Table Free Image}

This section is broken into two sub-sections. The first sub-section presents the table extraction processing in accordance with a modified reconstruction algorithm, while the second sub-section describes how to obtain a table free image based on Minkowski operation.

\subsection{Composing Table Structure Image by Modified Reconstruction Algorithm}

In order to extract the table structure and further to produce the table free image, the reconstruction algorithm has to be considered. Based on the wavelet theory, it is proved that no information is lost when an image is transformed into four sub-images. On the other hand, the original image can be reconstructed by these sub-images. The wavelet reconstruction is given by [5]. Based on the wavelet reconstruction algorithm, the original image can be reconstructed by

$$
\begin{aligned}
f\left(2 k_{1}+i_{1}, 2 k_{2}+i_{2}\right) & =\sum_{m_{1}=0}^{N-1} \sum_{m_{2}=0}^{N-1} h_{2 m_{1}+i_{1}} h_{2 m_{2}+i_{2}} f_{L L}\left(k_{1}-m_{1}, k_{2}-m_{2}\right) \\
& +\sum_{m_{1}=0}^{N-1} \sum_{m_{2}=0}^{N-1} h_{2 m_{1}+i_{1}} g_{2 m_{2}+i_{2}} f_{L H}\left(k_{1}-m_{1}, k_{2}-m_{2}\right) \\
& +\sum_{m_{1}=0}^{N-1} \sum_{m_{2}=0}^{N-1} g_{2 m_{1}+i_{1}} h_{2 m_{2}+i_{2}} f_{H L}\left(k_{1}-m_{1}, k_{2}-m_{2}\right) \\
& +\sum_{m_{1}=0}^{N-1} \sum_{m_{2}=0}^{N-1} g_{2 m_{1}+i_{1}} g_{2 m_{2}+i_{2}} f_{H H}\left(k_{1}-m_{1}, k_{2}-m_{2}\right)
\end{aligned}
$$

where $i_{1} \in\{0,1\}, i_{2} \in\{0,1\}$.

In this wavelet reconstruction algorithm, the reconstructed image will be very close to the original one if the value of $N$ is big enough for Daubechies wavelet. However, the results may meet the requirements of a particular application when $N$ is a small value because the line width of the table in the form document image is small. 


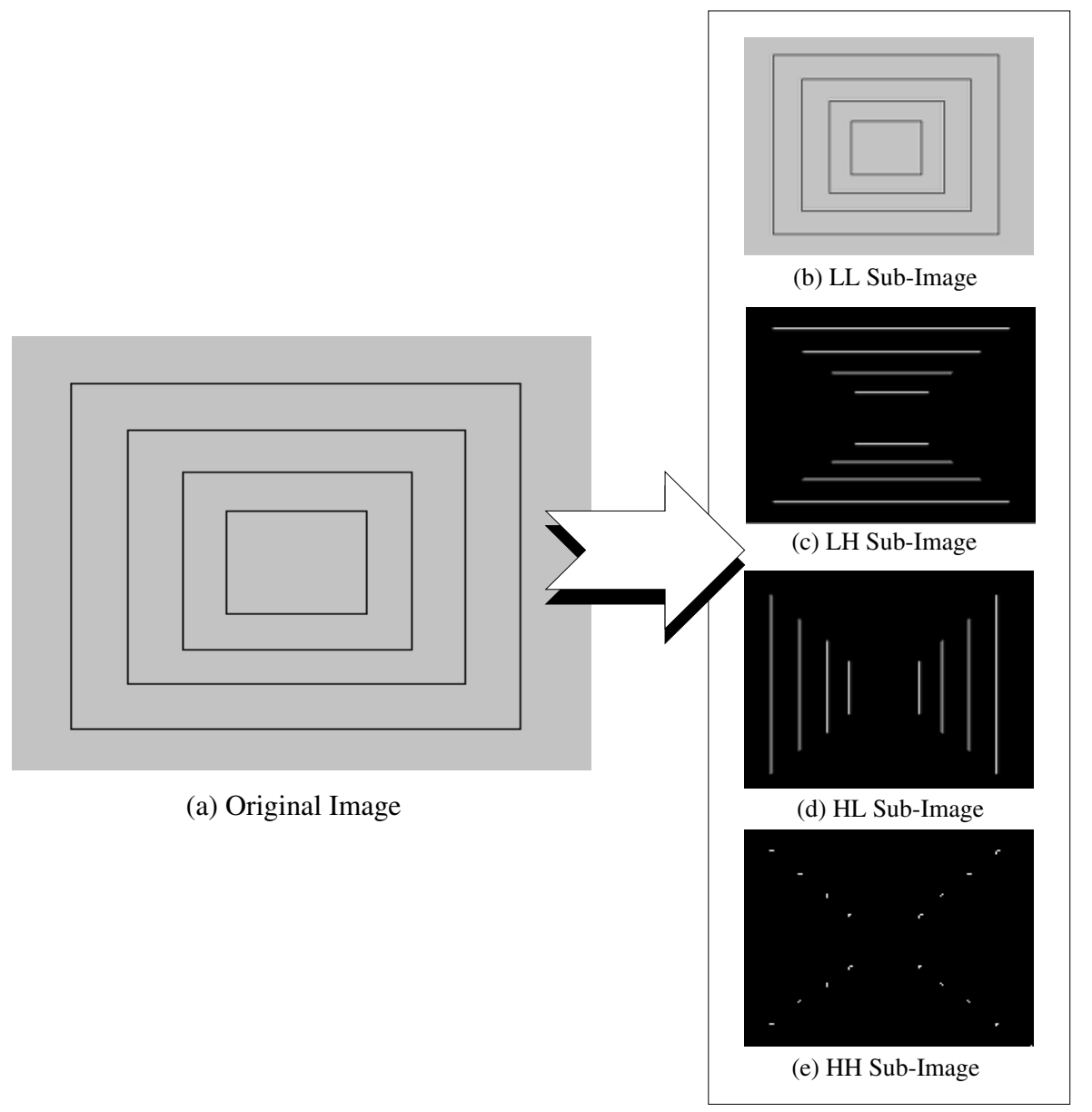

Fig. 3. Example of the decomposition of an image. (a) The original image with several nested squares. (b)-(e) The sub-images which are obtained from the original image by the wavelet decomposition algorithm.

To extract the table lines in a form document image, the components which contain only the horizontal and vertical lines have to be enhanced while other components have to be diminished. According to the analysis in the previous section, it is obvious that the horizontal lines can be enhanced by proliferating the LH sub-image component, while the vertical lines can be enhanced by proliferating the HL sub-image component. Therefore, it seems that the LH and HL sub-images can be used to reconstruct a table structure image which contains mainly table lines of the original image. However, such a table structure image may contain text-noise which has to be removed. Fortunately, the HH sub- 
image can be severed as a constraint condition to solve this problem, since it contains the main components of the text image. Based on this idea, the wavelet reconstruction algorithm has been modified to achieve the goal. The modified reconstruction algorithm is described as

$$
f_{\text {table }}(m, n)=\left\{\begin{array}{ll}
0 & \text { if } f_{\text {table }}^{\prime}(m, n)<0 \\
f_{\text {table }}^{\prime}(m, n) & \text { if } f_{\text {table }}^{\prime}(m, n) \geq 0
\end{array},\right.
$$

where

$$
\begin{aligned}
& f^{\prime}\left(2 k_{1}+i_{1}, 2 k_{2}+i_{2}\right) \\
& =C_{1} \sum_{m_{1}=0}^{N-1} \sum_{m_{2}=0}^{N-1} h_{2 m_{1}+i_{1}} g_{2 m_{2}+i_{2}} f_{L H}\left(k_{1}-m_{1}, k_{2}-m_{2}\right) \\
& +C_{2} \sum_{m_{1}=0}^{N-1} \sum_{m_{2}=0}^{N-1} g_{2 m_{1}+i_{1}} h_{2 m_{2}+i_{2}} f_{H L}\left(k_{1}-m_{1}, k_{2}-m_{2}\right) \\
& -C_{3} \sum_{m_{1}=0}^{N-1}\left|\sum_{m_{2}=0}^{N-1} g_{2 m_{1}+i_{1}} g_{2 m_{2}+i_{2}} f_{H H}\left(k_{1}-m_{1}, k_{2}-m_{2}\right)\right|
\end{aligned}
$$

such that $C_{1}, C_{2}$ and $C_{3}$ are coefficients, and they should satisfy the condition of $0<C_{1}<C_{3}, 0<C_{2}<C_{3}$.

The modified wavelet reconstruction algorithm is used to extract the lines from the form document image in our research. We have known that the LH and HL sub-images contain the horizontal and the vertical components respectively, while the $\mathrm{HH}$ sub-image contains other information which can be used to restrain the "noise" from the image when reconstructing the table structure image. Considering the fact that the magnitude in the $\mathrm{HH}$ sub-image is much more smaller than in the HL and LH sub-images, the parameters $C_{1}$ and $C_{2}$ should be much more smaller than $C_{3}$, and absolute is used in the third item in Eq. (13). On the other hand, the table line would be removed if $C_{3}$ is too big. Based on our experiments, $C_{3}$ can be chosen as 5 to 10 times as $C_{1}$ and $C_{2}$.

An example of the reconstruction of the table structure image using this modified reconstruction algorithm is illustrated in Fig. 4. The original image which contains texts and lines is shown in Fig. 4(a), the sub-images which were obtained from the original image by the wavelet decomposition are given in Fig. 4(b)-(e), and the table structure image generated by the modified wavelet reconstruction algorithm is given in Fig. 4(f).

\subsection{Constituting Table Free Image by Minkowski Subtraction}

To produce the table free image, the operation of Minkowski subtraction [6] is applied.

Let $A$ be the set denoting the square object with all its elements denoted by a two-dimensional variable $\alpha$. Next, let $B$ be the set containing one point and a two-dimensional variable $\beta$ used to describe this point. The Minkowski addition of two sets $A$ and $B$, given by the symbol $\oplus$, is

$$
A \oplus B=\left\{\mu \in R^{2}: \mu=\alpha+\beta, \alpha \in A, \beta \in B\right\} .
$$


The dual of the Minkowski addition is the Minkowski subtraction which is defined as

$$
A \ominus B=\left(A^{c} \oplus B^{c}\right)^{c} .
$$

Based on the Minkowski subtraction, the table free image can be generated from the original image and the table structure image. Let $D_{\text {original }}, D_{\text {table }}$, and $D_{\text {text }}$ be a original form document image, a table structure image and a table free image respectively. The table free image can be produced by

$$
D_{\text {text }}=D_{\text {original }} \ominus D_{\text {table }}
$$

In accordance with the Eq. (16), we can remove all table lines from the original image. Considering gray-levels of the image, we should replace the certain gray values with the points where the lines have been removed. In this paper, the average gray value of the neighboring points of the replaced pixel has been used.

For a point, $f(i, j)$, on the horizontal lines, the neighborhoods $f(i, j+\alpha)$, where $\alpha= \pm 1, \pm 2, \pm 3$ have been chosen, while, on the vertical lines, the neighborhoods $f(i+\beta, j)$, where $\beta= \pm 1, \pm 2, \pm 3$, have been used. Therefore, the replaced point should be

$$
f(i, j)= \begin{cases}\frac{1}{6} \sum_{\alpha= \pm 1,2,3} f(i, j+\alpha), & \text { if the point is on the horizontal line } \\ \frac{1}{6} \sum_{\alpha= \pm 1,2,3} f(i+\alpha, j), & \text { if the point is on the vertical line. }\end{cases}
$$

Fig. 4(g) shows an example of the table free image that was produced by the original image and the table structure image using the Minkowski subtraction.

\section{Experiments and Discussion}

Experiments have been conducted using a personal computer PC/Pentium II (333MHZ), and the programs have been written in Visual $\mathrm{C}^{++}$5.0. The form documents were entered into the system by an optical scanner and converted to a monochrome gray scale images( 8 bits per pixel). The resolution of digitization in our experiments was $300 \mathrm{DPI}$.

In our experiments, the gradient algorithm has been used to deskew the form document image, and the value of $N$ in Eq. (1) and Eq. (2) is 3. The Daubechies wavelet has been applied to decompose the form document images. The value of $N$ used in Eq. (10) is 3, i.e., the values of $\left\{h_{k}\right\}$ are listed below:

$$
\begin{aligned}
& \left\{h_{k}\right\}=\left\{\begin{array}{lll}
0.332670 & 0.714816 & 0.630880-0.027983
\end{array}\right. \\
& -0.1870340 .0308410 .032883-0.010597\} \text {. }
\end{aligned}
$$

Chinese bank form documents has been utilized in our experiments. Fig. 5 and Fig. 6 show the two main process steps in the gradient-wavelet scheme.

The gradient algorithm has been used to deskew the original image, Fig. 5(a). According to the Eq. (1), Eq. (2), and Eq. (3), the distribution histogram of the 


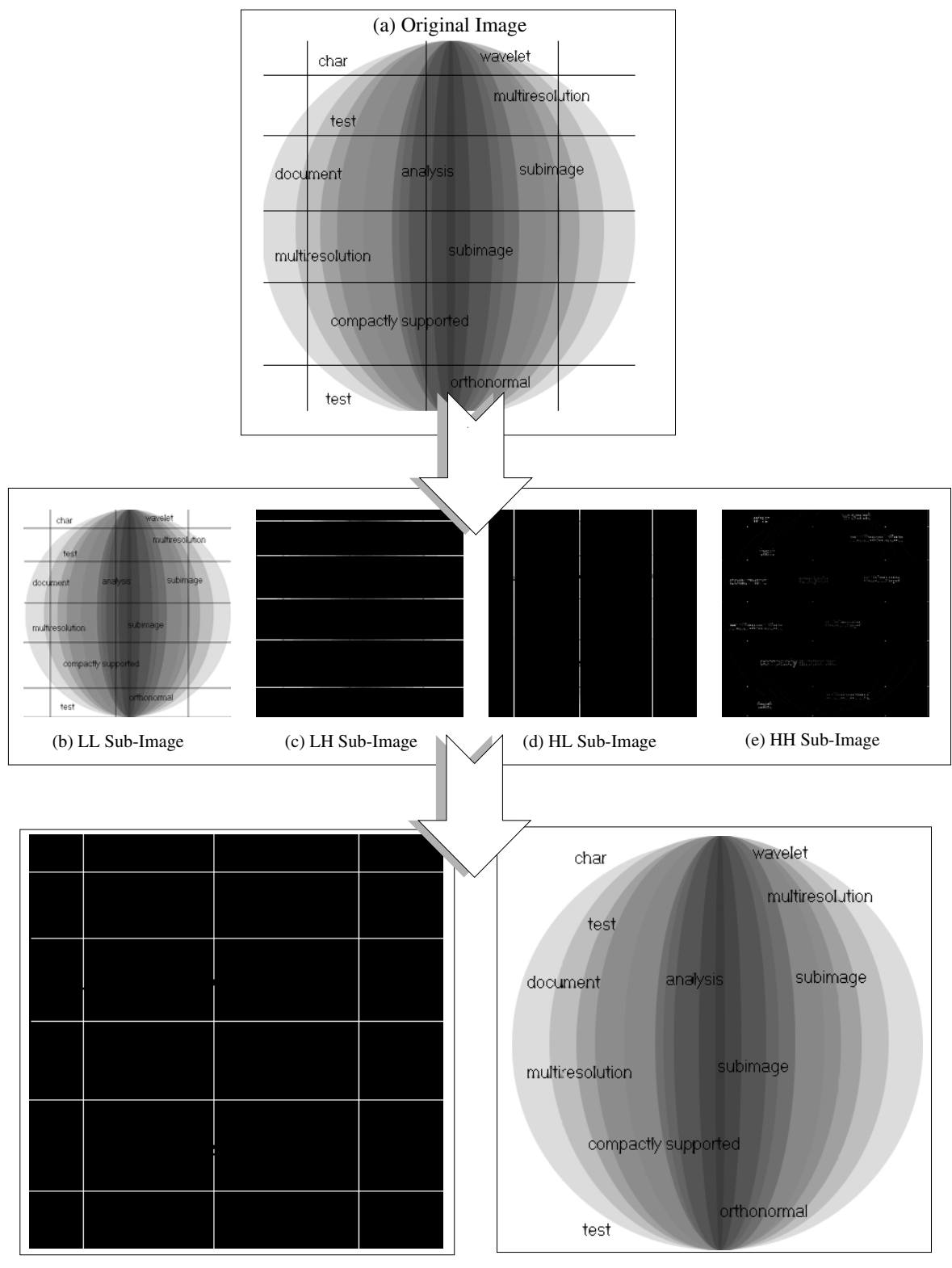

(f) Table-Structure Image

(g) Table-free Image

Fig. 4. Example of the wavelet decomposition and reconstruction. (a) The original image. (b)-(e) The sub-images obtained from the original image by the wavelet decomposition. (f) The table structure image generated by modified wavelet reconstruction algorithm. (g) The table free image produced by the original image and the table structure image. 

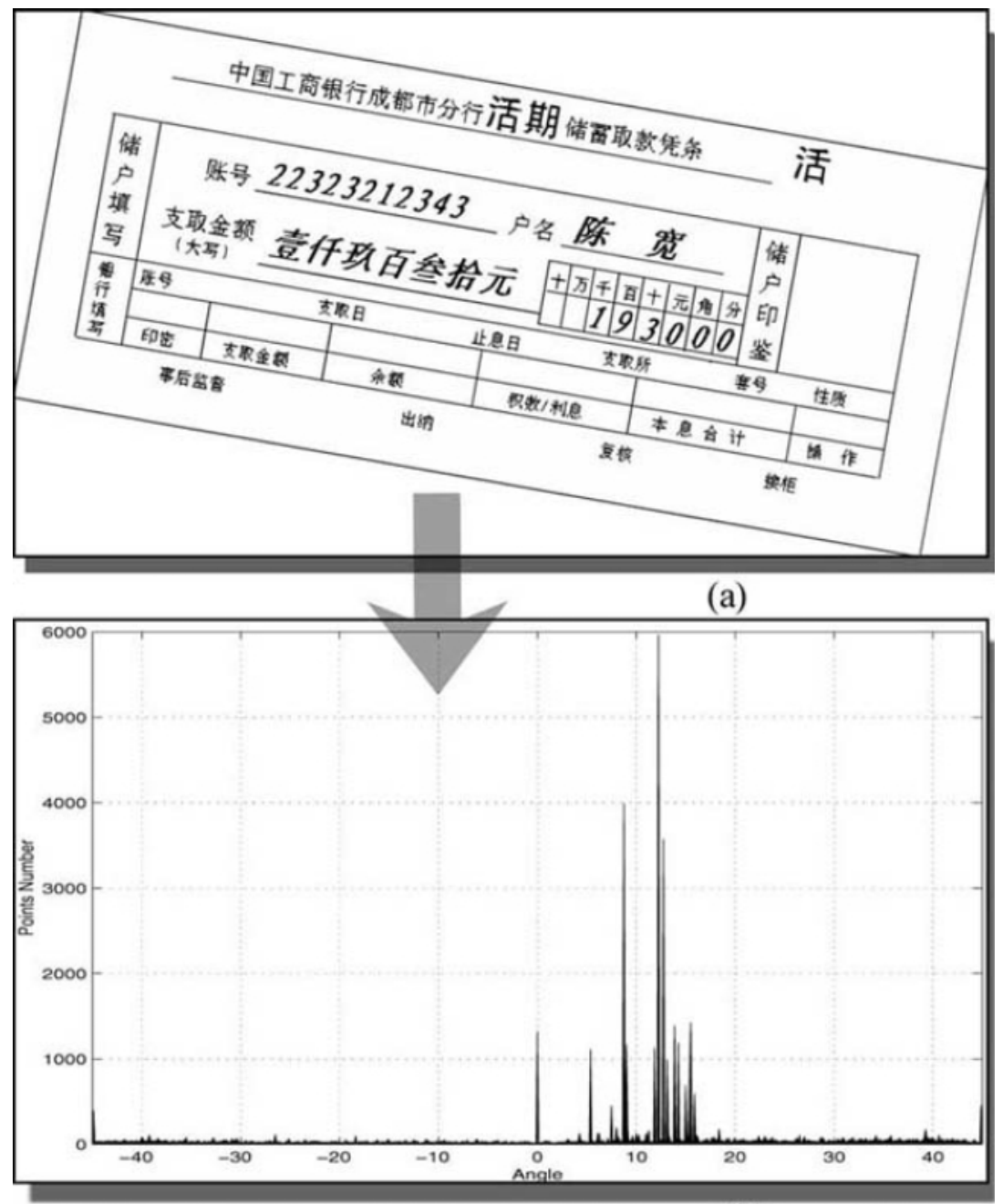

(b)

Fig. 5. The points distribution histogram obtained from gradient algorithm on different orientations for the Chinese bank document. (a) Original skewed form document image. (b) The distribution histogram on different gradient orientations of the original image.

original image on different orientation is shown in Fig. 5(b). The skew angle is the maximum point in the histogram, which was 12.5 in our example. The deskewed image obtained by Eq. (6) is shown in Fig. 6(a).

Wavelet decomposition and reconstruction of the Chinese bank form document image are illustrated in Fig. 6. The deskewed image is shown in Fig. 6(a), 
(a) Deskewed Image

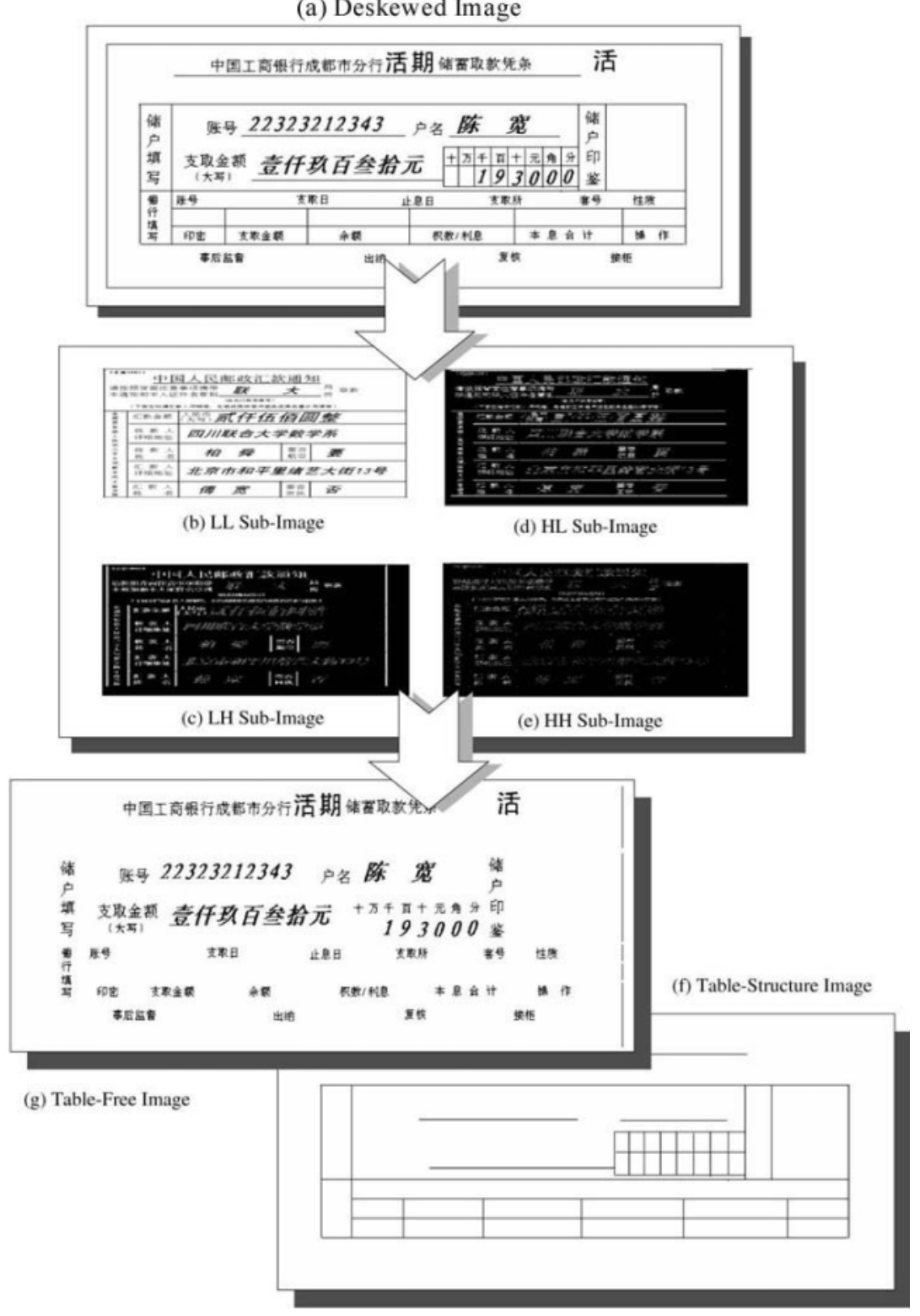

Fig. 6. Wavelet decomposition and reconstruction of Chinese bank document. (a) The deskewed image. (b)-(e) The sub-images obtained from the deskewed image by the wavelet decomposition. (f) The table structure image generated by modified wavelet reconstruction algorithm. (g) The table free image produced by the deskewed image and the table structure image. 
and its four wavelet sub-images are presented in Fig. 6(b)-(e), respectively. The table structure image generated by the modified wavelet reconstruction algorithm is given in Fig. 6(g). According to the table structure image and the deskewed image, the table free image can be constructed by the Minkowski subtraction, as shown in Fig. 6(f).

Processing of the form documents is an essential operation in many business and government organizations. Most of the significant information is located by the table structure. A lot of researchers have paid attention to the developments of many methods to solve this problem. A particularly important method to extract lines is Hough transform [8] which locates line in image through detecting the parameters of the line equation. Unfortunately, almost all techniques presented can not find the width and the end points of line exactly because they are based on the geometry.

\section{Acknowledgement}

This research was supported by the Hallym Academy of Sciences, Hallym University.

\section{References}

1. R. G. Casey, D. R. Ferguson, K. M. Mohiuddin, and E. Walach, "Intelligent Forms Processing System," Machine Vision and Application, Vol. 5, No. 3, pp. 143-155, 1992. 240

2. ICDAR'95, Proc. Third Int. Conf. on Document Analysis and Recognition, Montreal, Canada, August 14-16, 1995. 240

3. ICDAR'97. Proc. Fourth Int. Conf. on Document Analysis and Recognition, UlmGermany, August 18-20, 1997. 240

4. Y. Y. Tang, H. Ma, J. Liu, B. Li, and D. Xi, "Multiresolution Analysis in Extraction of Reference Lines from Documents with Gray Level Background," IEEE Trans. on Pattern Analysis and Machine Intelligence, Vol. 19, No. 8, pp. 921-926, 1997. 242, 245

5. S. Mallat, "A Theory of Multiresolution Signal Decomposition: the Wavelet Representation," IEEE Trans. on Pattern Analysis and Machine Intelligence, Vol. 11, pp. 674-693, 1989. 242, 247

6. R. Jain, "Extraction of Motion Information from Peripheral Processes," IEEE Trans. on Pattern Analysis and Machine Intelligence, Vol. 3, No. 5, pp. 489-503, 1981. 242, 249

7. S. Mallat, A Wavelet Tour of Signal Processing, San Diego: Academic Press, 1998.

8. E. Turolla, Y. Belaid, and A. Belaid. "Form Item Extraction Based on Line Searching", in Graphics Recognition: Method and Applications, Lecture Notes in Computer Science, Vol. 1072, Springer-Verlag, Berlin Heidelberg New York , pp. 69-79, 1996. 254 